\title{
Telemedicine-Based Tobacco Treatment Model in Primary Care from a Low-Resource Setting
}

\author{
Kumar Thamaraiselvan Santhosh ${ }^{1}$ Mani Bhushan Pant ${ }^{2}$ \\ C. Naveen Kumar ${ }^{4}$ Suresh Bada Math ${ }^{4}$ \\ ${ }^{1}$ Telemedicine Department of Psychiatry, National Institute of \\ Mental Health and Neuro Sciences (NIMHANS), Bengaluru, India \\ ${ }^{2}$ Community Health Centre, Gairsain, Chamoli District, \\ Uttarakhand, India \\ ${ }^{3}$ Non Communicable Disease Cell, National Health Mission, \\ Uttarakhand, India \\ ${ }^{4}$ Department of Psychiatry Primary Care Psychiatry Program, Tele \\ Medicine Centre, National Institute of Mental Health and Neuro \\ Sciences (NIMHANS), Bengaluru, India
}

Fareed Uzzafar ${ }^{3} \quad$ Narayana Manjunatha ${ }^{4}$

\begin{abstract}
Address for correspondence Narayana Manjunatha, MD, Department of Psychiatry, National Institute of Mental Health and Neuro Sciences (NIMHANS), Bengaluru 560029, Karnataka, India (e-mail: manjunatha.adc@gmail.com).
\end{abstract}

J Neurosci Rural Pract 2019;10:690-692

\begin{abstract}
Keywords

- tobacco

- nicotine

- addiction

- dependence

- primary care

- telemedicine

- India

Tobacco addiction is one of the leading causes of premature mortality. Early and effective intervention in primary care, though possible and feasible, is seldom done in India. This case series describes the diagnosis and management of three patients of tobacco addiction by a primary care doctor (PCD) who is being trained in digitally driven four modules based "Primary Care Psychiatry Program" of National Institute of Mental Health and Neurosciences, Bengaluru, India. This article discusses about the way in which two modules (telepsychiatric on-consultation training and collaborative video consultation) helped a primary care doctor (M.B.P.) working approximately 1,500 miles away from an academic institute to treat tobacco dependence at a rural primary health center of India.
\end{abstract}

\section{Introduction}

Tobacco addiction kills one in six smokers globally and one in two in low- and middle-income countries and remains a major public health concern. ${ }^{1,2}$ In India, $42.4 \%$ of men, $14.2 \%$ of women, and $28.6 \%$ ( 266.8 million) of all adults currently use tobacco (smoked and/or smokeless tobacco). ${ }^{3}$ Treatment gap for Tobacco use disorders is $91.8 \% .{ }^{4}$ Only way to address this huge gap is to integrate management of tobacco addiction into primary health care. ${ }^{4}$ Though this is shown to be possible, ${ }^{5}$ the ground reality is not encouraging. ${ }^{6}$

One recent effort by the National Institute of Mental Health and Neurosciences (NIMHANS), Bengaluru, India toward integrating mental health care into general health care is an innovative digital driven four module based primary care psychiatry programs (PCPPs). ${ }^{7}$ This involves mentoring the primary care doctors (PCDs) at their working clinic from an academic hospital using digital technology. ${ }^{7}$
An primary care adopted all-in-one training tool, namely "clinical schedules for primary care psychiatry" (CSP) is designed for this 1 year PCPP meant only for practicing PCDs. This CSP contains screener, primary care adopted taxonomy, diagnosis, treatment (with brief counseling), as well as follow-up guidelines for highly prevalent six psychiatric disorders at primary care one of which is tobacco addiction. ${ }^{8}$ CSP has been validated at primary care. ${ }^{9}$ A PCD who is working at primary health center of Uttarakhand state, India, had an opportunity to treat three patients with tobacco addiction with the help of two modules of PCPP, namely, the telepsychiatric on-consultation training (tele-OCT; a real time clinical training while the PCDs is in live consultation of his general patients in his/her clinic) and the collaborative video consultation (CVC: it is an videoconference based, on-demand, walk-in clinic for PCDs to obtain consultation) by a telepsychiatrist from NIMHANS, Bengaluru, an academic institute. CVC is provided to PCDs only after completing at least one 
session of tele-OCT and starts managing cases at the primary health centre (PHC), particularly for cases that PCD thinks expert help is required.

Authors describe the way two digital modules of PCPP from an academic institute based at Bengaluru, India, assisted a PCD who is working at approximately 1,600 miles away at a rural primary health center of Uttarakhand, India.

\section{Case Series}

\section{A. First Consultation in Tele-OCT Module}

Brief baseline clinical descriptions of three consecutive patients who consulted for tobacco use during their first contact with the PCD during tele-OCT sessions are described here.

A 35-year-old male presented with history of tobacco use in the form of chewing tobacco for past 10 years. He was chewing 7 to 8 packs of local tobacco per day. He reported to have increased intake in the last 3 to 4 years and had many attempts at abstinence. There were relapses of tobacco use due to severe craving (case 1). A 45-year-old male with history of tobacco use (beedi), at least 20 per day for past 12 years and alcohol use (once in a month). He reported that he had multiple attempts of failed abstinence of tobacco addiction (case 2). A 40-year-old male presented with history of alcohol and tobacco use for past 5 years. He reported to have a binge pattern of alcohol use and tobacco chewing (9-10 g/day) for the last 5 to 6 years. He reported that the last intake of alcohol was 4 months back and he didn't want help for his alcohol use. He wanted help for his tobacco chewing (case 3).

Above patients were screened with a single screener question "Do you use beedi/cigarettes/gutka or other tobacco products within 1 hour of getting up from bed in the early morning?" of CSP. Subsequent to "Yes" response, diagnosis of tobacco addiction was confirmed from the diagnostic guidelines provided in CSP. There was no history of epilepsy in all three patients. As per CSP's management guideline, bupropion 150 to $300 \mathrm{mg}$ after 5 days were prescribed with brief counseling for tobacco abstinence.

\section{B. Follow-up Consultations (using CVC Module)}

Follow-up consultations were done between 2 and 4 months after the initial consultation. The course and prognosis of these patients are briefed below.

Case 1 remained abstinent from tobacco with bupropion $300 \mathrm{mg} /$ day at 2 and 4 months of follow-up.

In case 2, at first follow-up (2 months), tobacco use reduced to 8 to 10 beedi/day on bupropion $300 \mathrm{mg} /$ day in 1 month, then relapsed to original tobacco use subsequent to stoppage of bupropion. It was discussed with him about the need for another trial of tablet bupropion, for which he agreed and medications restarted. At the end of 4 months in 2nd CVC follow-up, patient had decreased tobacco use (3-4 beedi/day) and continued medication use.

In case 3, at 2 months of follow-up, he had stopped tobacco chewing for 1 month and had restarted for the last 3 weeks despite on bupropion. The dose of bupropion was increased to $450 \mathrm{mg}$. At the end of 4 months of follow-up in CVC, patient stopped medications and restarted tobacco use. During follow-up, the patient was restarted with bupropion and subsequently lost to follow-up.

\section{Discussion}

This article demonstrates how a PCD can provide treatment for tobacco addiction in their busy rural PHCs using innovative training and consultation approach of PCPP. This article emphasizes that early assessment and intervention for tobacco addiction can be made at the primary-care level itself. This also prevents many complications secondary to tobacco use. Several other community-based models of care in different parts of India have shown the effectiveness of continued care in improving outcomes in alcohol and other substance use disorders. ${ }^{10-12}$

In our tele-psychiatric training module, an Indianized single-screening question of CSP is used, which is pragmatic in the setting of an Indian PHC, where busy clinics handle around 100 patients daily. Most of the times, doctors find it difficult to spend even 5 minutes and hence heavily rely on pharmacotherapy which is also acceptable to the patient. As seen above, case 1 clearly benefited by the management at the primary-care level and cases 2 and 3 had their initiation of treatment that paved the path for their abstinence. As we can observe, the course and outcome in a primary-care center is similar to a tertiary center or quaternary centres. ${ }^{10}$ The three different outcomes are (1) complete abstinence, (2) reduction in the quantity of use, and (3) relapse of tobacco use after abstinence for some period. Relapse is the part and parcel of the treatment process in tobacco use disorders and focusing on addressing the reasons for relapse would help in attaining early successful remission. Naturally, the outcome of all three patients is different and it is important that the PCDs understand this. However, we acknowledge that 4 months of outcome is a too short period to comment on the meaningful outcome of persons with tobacco use disorders. In this context, it may be pragmatic for the PCD to refer patients to higher centers (for further management) in case patients continued using tobacco in their third or fourth follow-ups.

\section{Conclusion}

To conclude, digitally driven two modules of tele-OCT and CVC of PCPP is found to be useful in helping a PCD to treat tobacco addiction in a rural primary health center of India. This model though requires further evaluation.

\section{Funding}

The authors acknowledge the funding of "National Health Mission" of Uttarakhand for funding this 1-year primary-care psychiatry program.

\section{Conflict of Interest}

None declared. 


\section{References}

1 WHO. WHO report on the Global Tobacco Epidemic 2009. Available at: https://apps.who.int/iris/bitstream/handle/ 10665/85380/9789241505871_eng.pdf;jsessionid=C9A67DC1170EC11DA8464EB50987455E? sequence $=1$. Accessed January 23, 2019

2 Parashar M, Agarwalla R, Mallik P, Dwivedi S, Patvagekar B, Pathak R. Prevalence and correlates of nicotine dependence among construction site workers: A cross-sectional study in Delhi. Lung India 2016;33(5):496-501

3 GATS2 (Global Adult Tobacco Survey) Fact Sheet, India, 2016-17 Available at: https://www.who.int/tobacco/surveillance/survey/gats/GATS_India_2016-17_FactSheet.pdf. Accessed October 14, 2019

4 [No authors listed]. What is primary care mental health?: WHO and Wonca working party on mental health. Ment Health Fam Med 2008;5(1):9-13

5 Kunyk D, Els C, Papadakis S, Selby P. Tobacco use disorder treatment in primary care: implementing a clinical system pathway in Alberta. Can Fam Physician 2014;60(7):646-655

6 Ziedonis DM. Barriers and solutions to addressing tobacco dependence in addiction treatment programs. 2006;29(3):8

7 Manjunatha N, Kumar CN, Math SB, Thirthalli J. Designing and implementing an innovative digitally driven primary care psychiatry program in India. Indian J Psychiatry 2018;60(2):236-244

8 Manjunatha N, Kumar CN, Math SB, Basvaraju V, Shashidhara HN, Parthasarathy P, et al. Karnataka Telemedicine, Mentoring and Monitoring Program: An implementation Manual of Primary Care Psychiatry, Bengaluru, National Institute of mental Health and Neuro Sciences, NIMHANS Publication No. 157, 2019 (ISBN: 978-81-86506-00-4)

9 Kulkarni K, Adarsha AM, Parthasarathy R, Philip M, Shashidhara HN, Vinay B, Manjunatha N, Kumar CN, Math SB, Thirthalli $\mathrm{J}$. Concurrent validity and inter-rater reliability of the clinical schedules for primary care psychiatry. J Neurosci Rural Pract 2019; doi:10.1055/s-0039-1697878

10 Chavan BS, Gupta N. Camp approach: a community-based treatment for substance dependence. Am J Addict 2004; 13(3):324-325

11 Kar N, Sengupta S, Sharma P, Rao G. Predictors of outcome following alcohol deaddiction treatment: a prospective longitudinal study for one year. Indian J Psychiatry 2003;45(3):174-177

12 Murthy P, Chand P, Harish M, et al. Outcome of alcohol dependence: the role of continued care. Indian J Community Med 2009;34(2):148-151 